\title{
Risk of Malignancy in a Nationwide Cohort of Elderly Inflammatory Bowel Disease Patients
}

\author{
Nabeel Khan ${ }^{1} \cdot$ Carlos Vallarino $^{2} \cdot$ Trevor Lissoos $^{3} \cdot$ Umar Darr $^{4}(\mathbb{D} \cdot$ \\ Michelle Luo ${ }^{3}$
}

Published online: 23 November 2017

(c) The Author(s) 2017. This article is an open access publication

\begin{abstract}
Background Management of elderly inflammatory bowel disease (IBD) patients ( $\geq 65$ years of age) is complicated due to many factors, including a higher risk of cancer, which may impact therapeutic decisions.

Objective The aim of this study was to determine the risk of cancer among elderly IBD patients compared with younger IBD patients. Additionally, the absolute risk of malignancy and factors contributing to it were evaluated, and therapeutic patterns among the elderly were assessed. Methods This retrospective cohort study extracted data from the Truven Health Analytics MarketScan ${ }^{\circledR}$ database. Among adult IBD patients who were free of cancer before starting on corticosteroids, immunomodulators, or biologics, a Cox model for time to cancer was fitted that adjusted for several covariates, including time-dependent treatment. Baseline results were evaluated by age group, as were the incidence of cancer and the distribution of cancer subtypes.
\end{abstract}

Nabeel Khan

nabeelk@mail.med.upenn.edu

1 Philadelphia VA Medical Center, University of Pennsylvania Perelman School of Medicine, Philadelphia, PA, USA

2 Data Science Institute, Takeda Development Center Americas, Deerfield, IL, USA

3 Takeda Pharmaceuticals USA, Inc, Deerfield, IL, USA

4 University of Toledo Medical Center, Toledo, OH, USA
Results The elderly IBD cohort $(n=8788)$ had a higher prevalence of cancer and several other ailments before starting treatment, relative to the younger IBD cohort aged 18-64 years $(n=54,971)$. During follow-up, the elderly IBD cohort experienced a higher incidence of malignancy, confirmed by a hazard ratio (HR) of 3.04 (95\% confidence interval [CI] 2.71-3.41) from the Cox model fit. The risk of cancer was also significantly associated with male sex (HR 0.82 female), duration of disease (HR 1.08), several comorbidities and corticosteroid use (HR 1.35), but not with the use of immunomodulators or biologics. NonHodgkin's lymphoma, urinary tract malignancy, and prostate, lung, and female breast cancers were observed more commonly in this elderly IBD cohort when compared with the same age group in the Surveillance, Epidemiology, and End Results (SEER) database.

Conclusions The elderly with IBD have a higher risk of malignancy when compared with younger IBD patients and the general age-matched population, with certain cancers being more common among these patients.

\section{Key Points}

Elderly patients with IBD have an increased risk of malignancy when compared with the general population.

This increased risk was associated with corticosteroid use, but not with immunomodulators or biologics.

The elderly are more likely to be treated with corticosteroids than corticosteroid-sparing agents. 


\section{Background}

Crohn's disease (CD) and ulcerative colitis (UC), collectively known as inflammatory bowel disease (IBD), affect nearly 1.6 million Americans [1]. The global increase in incidence and prevalence of IBD coupled with an aging population imply that the number of elderly patients with IBD is set to increase [2]. Management of elderly patients with IBD in the real world is complicated due to comorbidities, polypharmacy, and risk of adverse drug effects $[3,4]$.

Existing data have shown that chronic corticosteroid use is prevalent among elderly patients with IBD, with lower rates of utilization of corticosteroid-sparing regimens $[5,6]$. Many reasons are suspected for the underutilization of corticosteroid-sparing regimens in the elderly population $[7,8]$, including paucity of clinical data, partly due to the fact that clinical trials tend to exclude people over 65 years of age, as well as fear of infections secondary to immunosuppression. In particular, the elderly have an increased risk of malignancy as a factor of their age, which could be accentuated by the immunosuppressive medications used for the treatment of IBD $[8,9]$.

In view of the increasing number of elderly IBD patients and the lack of data regarding treatment patterns and safety profiles of medications in this cohort, we felt it essential to evaluate a nationwide cohort to gain further insight into this group. We designed this study to determine whether elderly IBD patients who are exposed to corticosteroids, immunomodulators, and biologic therapy have a significantly higher risk of cancer, even when controlling for several confounders, including treatment. As part of the study, we assessed the demographic features of elderly patients with IBD, as well as treatment modalities employed to manage the disease in this cohort, compared with younger adult patients (18-64 years of age). We also compared the incidence rates (IRs) and distribution of cancers among the elderly with age-matched controls, as determined by the Surveillance, Epidemiology, and End Results (SEER) database.

\section{Methods}

\subsection{Study Design}

This retrospective cohort study analyzed data between 1 January 2010 and 31 December 2014 from Truven Health Analytics MarketScan ${ }^{\circledR}$, a nationally representative US commercial claims and Medicare supplemental database consisting of medical and pharmacy claims of over 150 employers, including 100 health plans (payers), representing approximately 170 million covered lives.

\subsection{Patient Selection}

The study population consisted of patients with at least two International Classification of Diseases, Ninth Revision (ICD-9) diagnostic claims for UC (556.xx) or CD (555.xx), but not both, at least 30 days apart $(n=367,510$ included). After establishing the diagnosis, patients were followed starting on the date of the first prescription (index date) of a corticosteroid, immunomodulator, or biologic therapy (anti-tumor necrosis factor [TNF] or antiintegrin), from 2010 to 2014 ( $n=193,640$ excluded). To restrict our population to patients with moderate to severe IBD, 5-aminosalicylic acid was not included as an index therapy. All patients were $\geq 18$ years of age, had at least 6 months continuous enrollment prior to the index date (baseline period), and had at least 6 months of follow-up ( $n=78,944$ excluded). Patients with a diagnosis of rheumatoid arthritis or psoriasis during baseline were excluded ( $n=2252$ excluded), as were those with baseline claims for a biologic, immunomodulator, or corticosteroid ( $n=28,904$ excluded). An additional 11 patients were lost due to corrupted days' supply data, leaving the study with 63,759 evaluable subjects. To restrict our study to incident cancer, patients with at least one diagnosis of any malignancy during baseline $(n=3843)$ were excluded.

\subsection{Outcome Measures}

We defined a malignancy as a diagnosis with ICD-9 codes 140.xx-208.xx or 230.xx-234.xx (Table 5 in Appendix). The presence of cancer during follow-up was confirmed by requiring two identical ICD-9 codes on different dates within 2 months of each other [10]. Time to first cancer diagnosis was the primary outcome measure.

\subsection{Statistical Analysis}

The difference in the overall rates of malignancy between those aged 65 years or older and 18- to 64-year-olds was formally tested by focusing on the $p$ value for the age group hazard ratio (HR) in a Cox proportional hazards regression of time to first cancer. Variables adjusted for in the analytical model included IBD therapy (medication exposure) and all the baseline covariates listed in Table 1. A backward selection model was used and covariates with a $p$ value below 0.05 were retained, along with the age group indicator.

Medication exposure was defined in a time-dependent manner. The corticosteroid treatment variable was a simple yes/no indicator of ever use during follow-up. Immunomodulator and biologic treatment took on three possible values: 
Table 1 Baseline demographics and clinical characteristics

\begin{tabular}{|c|c|c|c|}
\hline Characteristic & Age $18-64$ years $[n=54,971]$ & $\begin{array}{l}\text { Age } \geq 65 \text { years } \\
{[n=8788]}\end{array}$ & $p$ Value \\
\hline Female & $31,037(56.5)$ & $4845(55.1)$ & 0.0197 \\
\hline $\mathrm{CD}$ & $24,929(45.3)$ & $3393(38.6)$ & $<0.0001$ \\
\hline $\mathrm{UC}$ & $30,042(54.7)$ & $5395(61.4)$ & \\
\hline \multicolumn{4}{|c|}{ Time from diagnosis to index, years } \\
\hline Mean & 1.14 & 1.42 & $<0.0001$ \\
\hline Median (SD) & $0.85(1.1)$ & $1.10(1.2)$ & \\
\hline \multicolumn{4}{|l|}{ Region } \\
\hline Northeast & $10,291(18.7)$ & 1895 (21.6) & $<0.0001$ \\
\hline Midwest & $13,872(25.2)$ & $2734(31.1)$ & \\
\hline South & $20,073(36.5)$ & $2488(28.3)$ & \\
\hline West & $8871(16.1)$ & $1587(18.1)$ & \\
\hline Unknown & $1864(3.4)$ & $84(1.0)$ & \\
\hline \multicolumn{4}{|l|}{ Type of health insurance } \\
\hline Comprehensive & $1714(3.1)$ & $3798(43.2)$ & $<0.0001$ \\
\hline HMO & $6062(11.0)$ & $966(11.0)$ & \\
\hline POS & $3257(5.9)$ & $281(3.2)$ & \\
\hline $\mathrm{PPO}$ & $33,790(61.5)$ & $3521(40.1)$ & \\
\hline Missing/unknown & $10,148(18.5)$ & $222(2.5)$ & \\
\hline \multicolumn{4}{|l|}{ Index year } \\
\hline 2010 & $13,449(24.5)$ & $2103(23.9)$ & 0.0203 \\
\hline 2011 & $13,691(24.9)$ & $2141(24.4)$ & \\
\hline 2012 & $11,681(21.2)$ & $1818(20.7)$ & \\
\hline 2013-2014 & $16,150(29.4)$ & $2726(31.0)$ & \\
\hline \multicolumn{4}{|l|}{ Comorbidities } \\
\hline Anemia & $5907(10.7)$ & $1436(16.3)$ & $<0.0001$ \\
\hline Cancer & $2393(4.4)$ & $1450(16.5)$ & $<0.0001$ \\
\hline COPD & $905(1.6)$ & 1005 (11.4) & $<0.0001$ \\
\hline Depression & $3687(6.7)$ & $493(5.6)$ & 0.0001 \\
\hline Diabetes & $3333(6.1)$ & $1689(19.2)$ & $<0.0001$ \\
\hline Hypertension & $9241(16.8)$ & $4379(49.8)$ & $<0.0001$ \\
\hline Ischemic heart disease & $1390(2.5)$ & $1655(18.8)$ & $<0.0001$ \\
\hline Osteoporosis & 885 (1.6) & $531(6.0)$ & $<0.0001$ \\
\hline Peripheral vascular disease & $254(0.5)$ & $453(5.2)$ & $<0.0001$ \\
\hline Solid organ transplant & $123(0.2)$ & $22(0.3)$ & 0.6271 \\
\hline \multicolumn{4}{|l|}{ Charlson comorbidity index } \\
\hline Mean score (SD) & $0.29(0.89)$ & $1.00(1.52)$ & $<0.0001$ \\
\hline Score $=0$ & $45,590(82.9)$ & $4720(53.7)$ & \\
\hline Score $\geq 1$ & $9381(17.1)$ & $4068(46.3)$ & \\
\hline \multicolumn{4}{|l|}{ Baseline medication } \\
\hline 5-ASA & $25,481(46.4)$ & 4528 (51.5) & $<0.0001$ \\
\hline \multicolumn{4}{|l|}{ Polypharmacy } \\
\hline Mean (SD) & $3.0(2.3)$ & $4.8(2.5)$ & $<0.0001$ \\
\hline $0-4$ medications & $41,775(76.0)$ & $4062(46.2)$ & \\
\hline $5+$ medications & $13,196(24.0)$ & $4726(53.8)$ & \\
\hline
\end{tabular}

Data are expressed as $n(\%)$ unless otherwise specified

5-ASA 5-aminosalicylic acid, CD Crohn's disease, COPD chronic obstructive pulmonary disease, $H M O$ health maintenance organization, $P O S$ point-of-service, $P P O$ preferred provider organization, $S D$ standard deviation, $U C$ ulcerative colitis 
- pre-treatment, from index date until first prescription (or never treated);

- on treatment, from first prescription of active course of therapy until 6 months after last day on drug;

- post-treatment, from 6 months after last day on drug until end of study, or until first prescription of a subsequent course of therapy.

This treatment scheme was implemented through two time-dependent indicator variables:

- on treatment versus other (pre- and post-treatment);

- post-treatment versus other (pre-treatment and on treatment).

Descriptive statistics were computed stratified by age group. Baseline cohort comparisons were based on $p$ values derived from a Chi-squared test for categorical variables and analysis of variance for continuous variables.

\subsection{Post Hoc Analysis}

After the results of the formal hypothesis test, we investigated the IRs and frequencies of certain types of cancer, by age group, and compared them with SEER statistics on the general population. Event rates by treatment exposure intervals were also explored.

\section{Results}

\subsection{Patient Characteristics}

The final cohort of 63,759 consisted of 54,971 patients between 18 and 64 years of age, and 8788 patients aged 65 years or older at index date. Table 1 displays the baseline characteristics of the population by age group. While the older and younger cohorts are similar in sex, index year and depression, there are some differences in region, health insurance and other variables. While some of the differences appear to be minimal, e.g. sex, the $p$ value for the difference between the two cohorts, driven by the large sample sizes, is very small.

Overall, the older age group had a larger percentage of patients with UC, more subjects from the Midwest and less from the South, more people with comprehensive health insurance, and more patients with chronic obstructive pulmonary disease (COPD), anemia, ischemic heart disease, cancer, diabetes and hypertension. Additionally, the older cohort had an average Charlson comorbidity index (CCI) score approximately 3.5-fold larger than the younger cohort, and used 1.5-fold more different types of medications (polypharmacy), painting a picture of an obviously sicker population compared with the younger group.
At baseline, 5-aminosalicylic acid use was higher among the elderly $(51.5 \%)$ compared with 18 - to 64-yearolds (46.4\%); this difference was driven by CD patients (43.4 vs. $33.0 \%, p<0.0001$ ), whereas 5-aminosalicylic acid use was balanced among UC patients (56.7 vs. $57.4 \%$, $p=0.29$ )

The median length of follow-up was 24 months for the elderly and 22 months for the younger group. From Table 2, we see that corticosteroid use any time during follow-up was higher among the elderly (96\%) compared with 18 - to 64-year-olds (90\%), while the reverse was true for utilization rates of biologics (6 vs. 19\%) and immunomodulators (11 vs. 21\%). Among biologics users, only 3 elderly patients $(0.6 \%)$ and 72 younger patients $(0.7 \%)$ were ever prescribed an anti-integrin (note that vedolizumab was licensed in May 2014 and natalizumab is seldom used for IBD therapy).

\subsection{Cancer Incidence}

For the analysis phase, the 1450 older patients and 2393 younger patients who had cancer at baseline (see Table 1) were excluded. The older cohort experienced a much higher incidence of malignancy. Based on 564 events (not including non-melanoma skin cancer [NMSC] events) during 15,824 person-years (PYs), patients over 65 years of age had an average IR of 3.56 cancers per $100 \mathrm{PYs}$, with a $95 \%$ confidence interval (CI) of 3.28-3.87. The elderly rate was almost fourfold the 0.93 rate for patients under 65 years of age (95\% CI 0.87-0.99), based on 1017 events

Table 2 Therapy any time during follow-up by IBD type and age groups

\begin{tabular}{llll}
\hline Therapy $^{\mathrm{a}}$ & Age 18-64 years & Age $\geq 65$ years & $p$ Value \\
\hline All patients & $n=54,971$ & $n=8788$ & \\
Corticosteroid & $49,592(90.2)$ & $8410(95.7)$ & $<0.0001$ \\
Immunomodulator & $11,374(20.7)$ & $960(10.9)$ & $<0.0001$ \\
Biologic & $10,326(18.8)$ & $519(5.9)$ & $<0.0001$ \\
UC patients & $n=30,042$ & $n=5395$ & \\
Corticosteroid & $28,459(94.7)$ & $5258(97.5)$ & $<0.0001$ \\
Immunomodulator & $5008(16.7)$ & $489(9.1)$ & $<0.0001$ \\
Biologic & $3151(10.5)$ & $196(3.6)$ & $<0.0001$ \\
CD patients & $n=24,929$ & $n=3393$ & \\
Corticosteroid & $21,133(84.8)$ & $3152(92.9)$ & $<0.0001$ \\
Immunomodulator & $6366(25.5)$ & $471(13.9)$ & $<0.0001$ \\
Biologic & $7175(28.8)$ & $323(9.5)$ & $<0.0001$ \\
\hline
\end{tabular}

Data are expressed as $n(\%)$

$C D$ Crohn's disease, $I B D$ inflammatory bowel disease, $U C$ ulcerative colitis

a Table represents cumulative drug use during follow-up, therefore each row is not mutually exclusive 
during 109,472 PYs (again, excluding cases of NMSC). Compared with the SEER database's age-specific cancer rates, IBD patients $<65$ years of age were exposed to a risk of cancer that was fourfold higher than the general population's rate of 0.22 cancers per $100 \mathrm{PYs}$, while IBD patients $\geq 65$ years of age were exposed to a cancer risk approximately 1.8 -fold higher than the SEER rate of 2.03 [11].

The hazard function, which is the instantaneous risk of an outcome (displayed in Fig. 1), essentially shows the progress of the incidence of cancer over time. The instantaneous risk of cancer was highest right after the index date but then slowly tapered off. The local peaks of both incidences near the 5-year mark were driven by relatively small sample sizes (see Fig. 1).

\subsection{Formal Cox Model Fit}

Confirming the difference seen in the raw IRs, the Cox model produced an HR of 3.04 for the over 65 years age group, with a $p$ value below 0.0001 , meaning that, in this study, the older cohort had an approximately threefold higher risk of cancer (based on time to the first cancer diagnosis) than the younger cohort, a highly statistically significant difference (Table 3). Several other baseline characteristics that were associated with an increased risk of cancer are reported in Table 3: male sex, time since the IBD diagnosis, taking at least five different types of medications (polypharmacy), a CCI score $\geq 1$, a solid organ transplant, hypertension, and COPD. Taking corticosteroids any time during follow-up was associated with a $35 \%$ increased risk of cancer. The correlations among the parameter estimates were all below 0.25 (absolute value), an indication of a well-determined model. No statistically significant association was observed between length of exposure to immunomodulators or biologics and cancer.

To produce the graphic showing the separation between the Kaplan-Meier survivor functions or event-free probability curves for each age group, a Cox model was fitted without the time-dependent treatment variables (Fig. 2).

A similar picture emerged for NMSC, analyzed as an experimental endpoint. The older cohort endured 529 first events and a raw IR of 3.36 cancers per 100 PYs, approximately fivefold higher than the 0.64 rate for the younger cohort, which was based on 700 events. However, a different set of significant covariates appeared in the proportional hazards model for NMSC. The HR for the over 65 years age group was 4.81 , with a $95 \%$ CI of $4.17-5.55$ and a $p$ value below 0.0001 . The time-dependent indicator of post-biologic treatment remained in the final model, associated with a $43 \%$ reduction in skin cancer risk, while the other three time-dependent indicators were dropped. Taking corticosteroids any time during follow-up was associated with a $30 \%$ decreased risk of NMSC. Other baseline characteristics that significantly altered the risk of cancer were male sex (HR 1.54), polypharmacy (HR 1.24), time since the IBD diagnosis (HR 1.07), a solid organ transplant (HR 3.62), region, and health insurance. Region yielded sensible results, with patients in the South and West (the sunnier areas of the country) exhibiting a higher
Fig. 1 Estimated hazard rate or incidence density of cancer over time with confidence bands

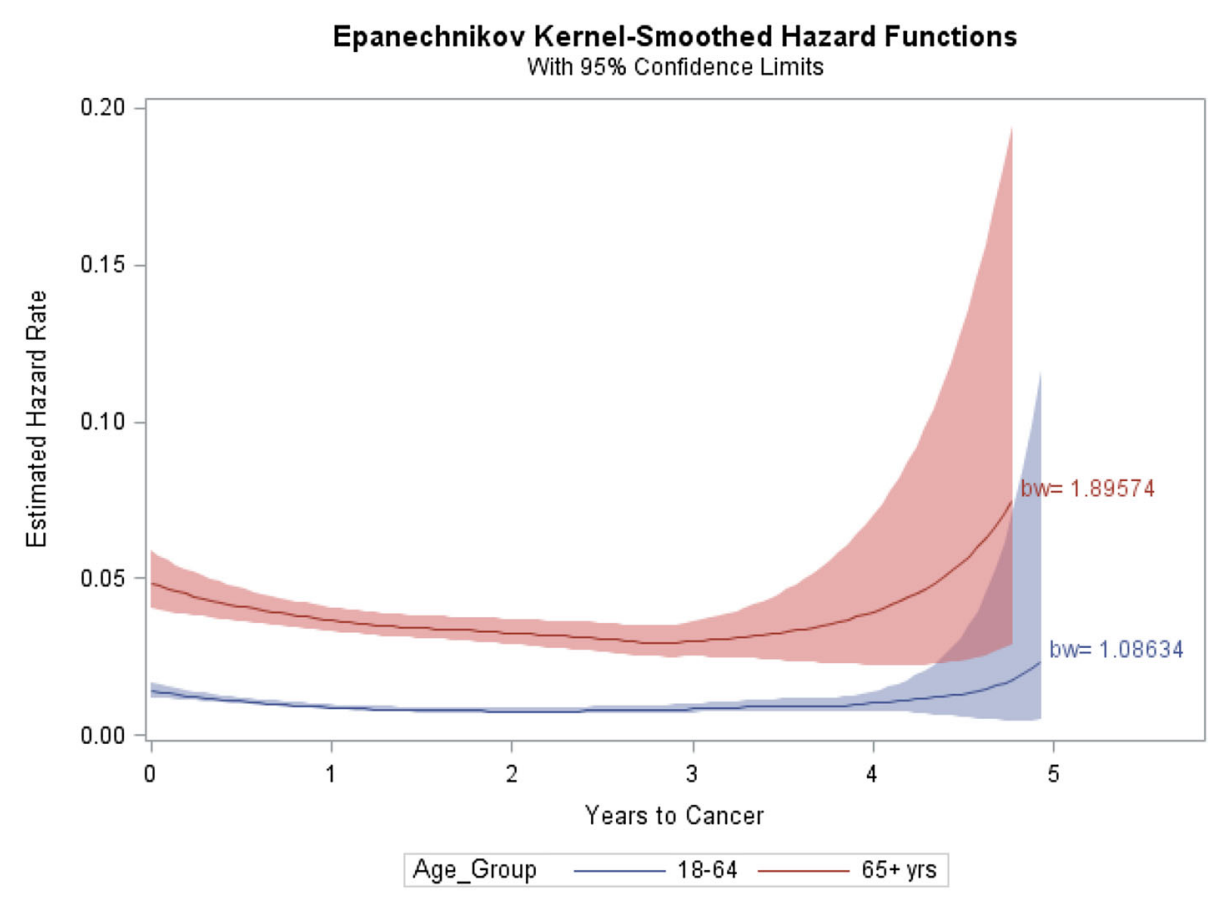

Note: Higher hazard rate belongs to elderly subgroup 
Fig. 2 Kaplan-Meier eventfree probability curves with confidence bands
Survivor Functions

With $95 \%$ Confidence Limits

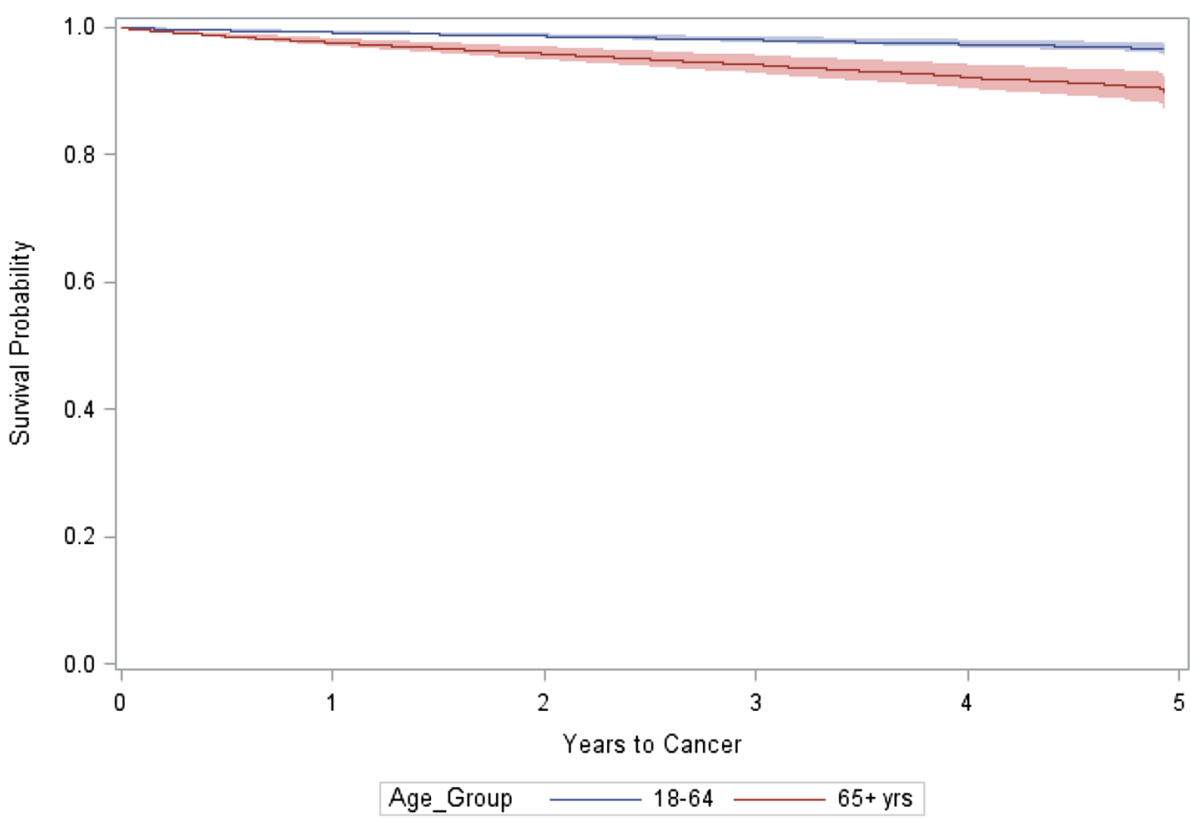

Table 3 Proportional hazards model of time to cancer: hazard ratios of significant covariates

\begin{tabular}{lllr}
\hline Parameter & Label & HR $(95 \%$ CI $)$ & $p$ Value \\
\hline Age group & $\geq 65$ years & $3.04(2.71-3.41)$ & $<0.0001$ \\
DISTIME & Dx to index date & $1.08(1.03-1.13)$ & 0.0020 \\
Corticosteroid & Corticosteroids any time & $1.35(1.08-1.69)$ & 0.0088 \\
Sex & Female & $0.82(0.74-0.90)$ & $<0.0001$ \\
COPD & Yes & $1.59(1.30-1.94)$ & $<0.0001$ \\
Solid organ transplant & Yes & $2.72(1.40-5.27)$ & 0.0031 \\
Charlson comorbidity index & $\geq 1$ Charlson score & $1.18(1.04-1.33)$ & 0.0107 \\
Hypertension & Yes & $1.15(1.02-1.30)$ & 0.0185 \\
Polypharmacy & $\geq 5$ medications & $1.22(1.09-1.37)$ & 0.0006 \\
\hline
\end{tabular}

CI confidence interval, COPD chronic obstructive pulmonary disease, DISTIME time from diagnosis to index date, $D x$ diagnosis, $H R$ hazard ratio risk of skin cancer than the reference region, the Midwest, or the Northeast.

\subsection{Post Hoc Analysis}

Following the formal hypothesis test, we explored the distribution of the types of malignancy in our study (Table 4), as well as the specific timing of the events relative to immunomodulator and biologic exposure. Overall, 1581 cancer events were observed in the study, by age group and level of the time-dependent treatment covariates. The raw average IRs of malignancy are displayed in Fig. 3a, b. The unadjusted IRs of cancer are higher across the pre-treatment, on-treatment, and post-treatment periods in IBD patients aged $>65$ years in the biologic- and immunomodulator-treated groups. With one exception, it is striking how similar the IRs are across the three time categories: pre-treatment (or no treatment), on treatment, and post-treatment. The only exception appears to be older patients during biologics post-treatment, although its wide CI reflects a reduced sample size. The rates for immunomodulator post-treatment also appear to be relatively lower than the other time frames.

Higher incidences of colorectal cancer (IR 0.27/100 PY vs. $0.20 / 100 \mathrm{PY}$ ), non-Hodgkin's lymphoma (IR 0.14/100 PY vs. 0.09/100 PY), and urinary tract malignancy (IR $0.37 / 100$ PY vs. $0.12 / 100 \mathrm{PY}$ ) were observed among IBD patients $\geq 65$ years of age when compared with cancer IRs in the SEER database for the same age group. Prostate, lung, and female breast cancers also had higher IRs in the elderly IBD subgroup compared with the age-matched SEER database. 
Table 4 Distribution of specific cancers among the elderly and younger age groups

\begin{tabular}{|c|c|c|}
\hline Cancer $^{\mathrm{a}}$ & Age $18-64$ years $[n=52,578 ; 1717$ events $]$ & Age $\geq 65$ years $[n=7338 ; 1093$ events $]$ \\
\hline \multicolumn{3}{|c|}{ GI malignancies with an increased incidence in IBD } \\
\hline Colorectal cancer & $111(6.5)$ & $43(3.9)$ \\
\hline Small bowel adenocarcinoma & $14(0.8)$ & $5(0.5)$ \\
\hline Anal cancer & $5(0.3)$ & $2(0.2)$ \\
\hline Cholangiocarcinoma & $8(0.5)$ & $1(0.1)$ \\
\hline \multicolumn{3}{|c|}{ Cancers possibly related to IBD medications } \\
\hline Urinary tract malignancy & $83(4.8)$ & $58(5.3)$ \\
\hline Melanoma & $94(5.5)$ & $24(2.2)$ \\
\hline NMSC & $700(40.8)$ & $529(48.4)$ \\
\hline Non-Hodgkin's disease & $43(2.5)$ & $22(2.0)$ \\
\hline \multicolumn{3}{|l|}{ Other cancers unrelated to IBD } \\
\hline Prostate cancer & $104(6.1)$ & $100(9.1)$ \\
\hline Female breast cancer & $164(9.6)$ & $77(7.0)$ \\
\hline Lung cancer & $32(1.9)$ & $72(6.6)$ \\
\hline Pancreatic cancer & $17(1.0)$ & $27(2.5)$ \\
\hline Acute myeloid leukemia & $7(0.4)$ & $3(0.3)$ \\
\hline Others $^{\mathrm{b}}$ & $335(19.5)$ & $130(11.9)$ \\
\hline
\end{tabular}

Data are expressed as number of events $(\%)$

GI gastrointestinal, IBD inflammatory bowel disease, NMSC non-melanoma skin cancer

${ }^{a}$ Percentage of each cancer subtype in the first event of cancer, e.g. colorectal cancer made up $6.5 \%$ of all first cancer events in the younger group, and $3.9 \%$ in the elderly group, ordered from the highest incidence in the $\geq 65$ years age group to the lowest

${ }^{\mathrm{b}}$ All other cancers, comprised of over 120 specific subtypes

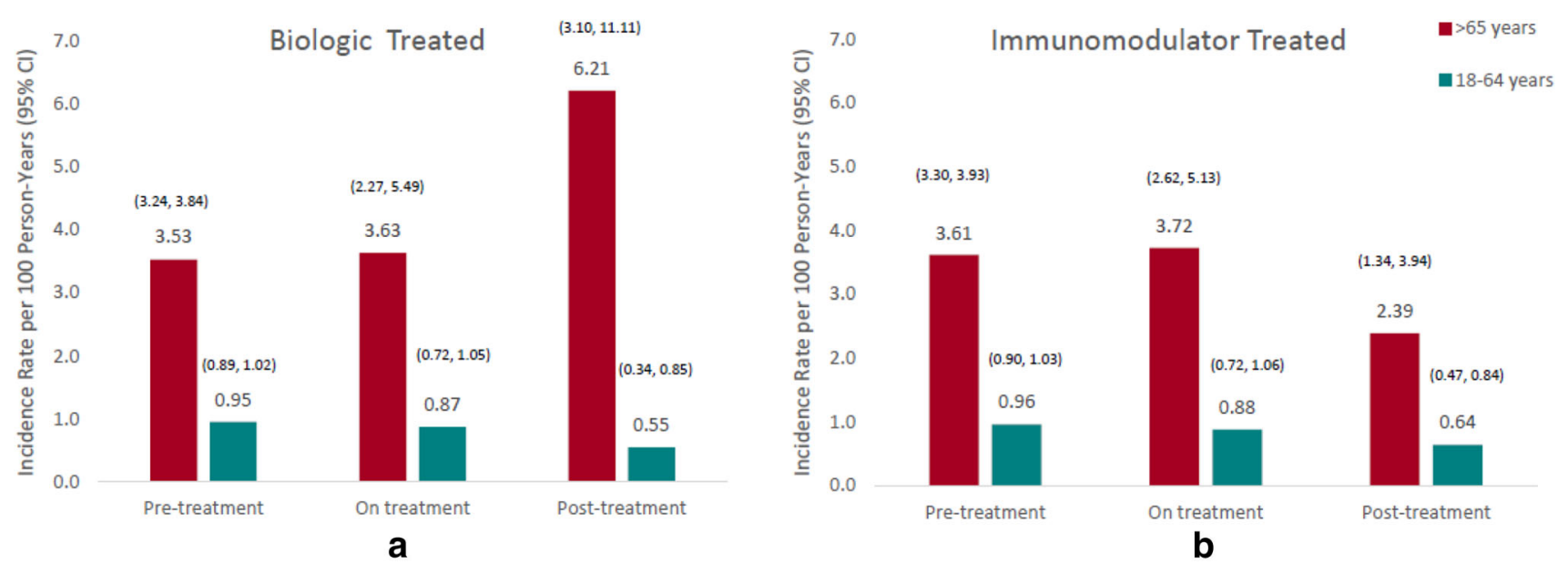

Fig. 3 Unadjusted incidence rates of cancer by exposure intervals of treatment with (a) biologics and (b) immunomodulators. $C I$ confidence interval

\section{Discussion}

The prevalence of IBD within an already aging population is increasing, yet management remains controversial due to factors such as comorbidity, polypharmacy, and adverse drug effects [3]. From this large, population-based, retrospective cohort study, we concluded that elderly IBD patients had a statistically significant threefold higher incidence of cancer compared with younger IBD patients, after controlling for time-dependent medications and a variety of baseline characteristics and comorbidities. Additionally, both the elderly and younger IBD cohorts had a higher risk of malignancy when compared with the agematched general population in the SEER database. 
While evaluating the demographic attributes of the elderly and younger IBD populations, we discovered that the older age group had a higher proportion of patients with UC, and, as expected, a higher prevalence of serious comorbidities. Almost half the patients (46.3\%) had a CCI of one or more. Polypharmacy was of particular concern in this elderly population, with more than half $(53.8 \%)$ taking five or more medications [12]. These data provide clinicians with a clearer understanding of their older IBD patients.

Interestingly, in regard to IBD-specific therapy, there are unique patterns in the use of corticosteroids, immunomodulators, and biologic agents. Corticosteroid use was marginally higher among the elderly (95.7 vs. $90.2 \%$ ), while there was significantly lower use of immunomodulators (10.9 vs. 20.7\%) and biologic agents (5.9 vs. $18.8 \%$ ). This implies that utilization of corticosteroid-sparing drugs is much lower among the elderly. Another interesting fact was the higher use of 5-aminosalicylic acid compounds among the elderly compared with the younger Crohn's patients, despite the lack of data demonstrating their efficacy in $\mathrm{CD}$, suggesting physicians are reluctant to use immunomodulators or biologics in the elderly. This lower use of corticosteroid-sparing agents in the elderly could possibly be explained by underrepresentation in clinical trials, minimal evidence-based literature, or physician concern for side effects associated with these classes of medications [13-16]. Foremost among these is the concern for malignancy. To address this issue we evaluated the absolute risk of malignancy and the factors associated with the development of malignancy.

In our population, older IBD patients experienced a much higher rate of malignancy, with an average raw IR of 3.56 cancers per 100 PYs. The cancer rates in the older IBD population were also higher than age-matched groups in the SEER database, which is representative of the general population. This was a significant finding in the study and although the cause is uncertain, it may be secondary to immune dysregulation that is inherent in IBD patients. Baseline characteristics that were associated with an increased risk of cancer included male sex, time since IBD diagnosis, polypharmacy use ( $\geq 5$ medications), a CCI score of at least 1 , solid organ transplant, hypertension, and COPD (refer to Table 3). Notably, corticosteroid use at any time was associated with a $35 \%$ increased risk of developing a malignancy.

Evaluating the risk associated with immunomodulators and biologics, there were similar IRs across the three categories of treatment: pre-treatment (or no treatment), on treatment and post-treatment. Our results collectively show that immunomodulators and biological agents tended not to increase the overall cancer incidence in this IBD population, in line with a previous study, which concluded that adalimumab did not increase the overall risk of malignancy [17], as well as data from the TREAT registry [18]. Similarly, a Danish study found that exposure to anti-TNF agents was not associated with an increased risk of cancer [19].

A prominent finding was that the elderly had a very high prevalence of baseline malignancy (16.5\%), which, coupled with a higher propensity for developing a malignancy in the follow-up period, places them at a high risk of developing cancer during the course of their disease. There are limited data on the use of thiopurines and anti-TNF agents among patients who have had a malignancy. A recent meta-analysis suggested that there was not an increased risk of recurrence of malignancy in this group [20]. However, in the absence of prospective data, judicious use of these therapies, or therapy with a gut selective mechanism of action, could be considered [21-24].

We also evaluated the distribution of specific cancer subtypes between the elderly and younger age groups. Among the elderly, the most common malignancies were prostate, lung, female breast cancer, urinary tract cancer, and colorectal cancer. While it is expected that higher rates of colorectal cancer would be seen in IBD patients, other malignancies such as non-Hodgkin's lymphoma, urinary tract malignancy, and prostate, lung, and female breast cancers were observed more commonly among IBD patients $\geq 65$ years of age when compared with the same age group in the SEER database [25]. Physicians caring for elderly IBD patients need to be cognizant of the increased risk of malignancy in this group, and should ensure that adequate screening modalities are utilized and patients made aware of early warning symptoms [16, 26].

This study is not without its limitations. The entire MarketScan ${ }^{\circledR}$ database contains longitudinal health claims from almost 170 million participants who were covered by a variety of health insurance plans, including a large number with Medicare supplemental policies. Of course, the database consists only of insured individuals, and does not represent the US population at large; its lack of direct Medicare patients and other non-commercially insured individuals entails a limitation of the database and therefore the study. The study was also limited by the unavailability of some confounders, such as race and smoking status, and by having only a partial measure of the length of time patients had lived with IBD before entering the study. The fact that this covariate still exerted so much influence on the fitted model speaks to its importance. Observational studies are, by their very nature, limited as lack of randomization introduces bias in the unbalanced selection of cohorts, although the large number of potential confounders in the model helped ameliorate the situation. It can be argued that cancer develops over many years, and hence the 6-month baseline and average follow-up of 
approximately 2 years may have been too short to fully assess the risk of malignancy. Although no statistically significant association was observed between the length of exposure to medications and the risk of cancer, these results should be interpreted with caution as the median duration of follow-up was only 22 months for the younger group and 24 months for the elderly, which may be too short to show an effect. As it was our intention to evaluate patients with moderate to severe IBD, we did not include in our analysis patients who were only treated with 5-aminosalicylic acid compounds, which led to the exclusion of a large proportion of patients.

\section{Conclusions}

Elderly IBD patients are a distinct group with increased comorbidities and polypharmacy. They have a substantially increased risk of malignancy when compared with both the younger IBD group and the general population. This is not just limited to a higher overall malignancy rate as certain malignancies are more common in the elderly IBD population. The increased rate was associated with corticosteroid use, but not with the use of biologics or immunomodulators. Despite this lack of association, elderly patients were more likely to be treated with corticosteroids than corticosteroid-sparing agents. Further studies should be performed among elderly IBD patients to assess the impact of longer-term treatment on cancer risk.
Author Contributions Nabeel Khan is overall guarantor of this article. Nabeel Khan, Carlos Vallarino, Trevor Lissoos and Michelle Luo contributed to study design, interpretation of data, and drafting, revision, and approval of the manuscript. Umar Darr contributed to drafting, revision, and approval of the manuscript. All authors approved the final version of the submitted manuscript.

\section{Compliance with Ethical Standards}

Conflicts of Interest Carlos Vallarino, Trevor Lissoos, and Michelle Luo are employees of Takeda Pharmaceuticals, USA. Nabeel Khan has received grant support from Pfizer, Takeda, and Luitpold Pharmaceuticals. Umar Darr has no declaration of conflicts of interest.

Funding This study was funded by Takeda Pharmaceuticals, USA. The authors gratefully acknowledge the participation of Sreevalsa Unniachan, $\mathrm{PhD}$, in the project, and the editorial assistance of Kyle Null, $\mathrm{PhD}$, in preparing this manuscript.

Open Access This article is distributed under the terms of the Creative Commons Attribution-NonCommercial 4.0 International License (http://creativecommons.org/licenses/by-nc/4.0/), which permits any noncommercial use, distribution, and reproduction in any medium, provided you give appropriate credit to the original author(s) and the source, provide a link to the Creative Commons license, and indicate if changes were made.

\section{Appendix}

See Table 5.
Table 5 Malignancy diagnosis codes

\begin{tabular}{lc}
\hline Malignancy & ICD-9 code \\
\hline Malignant neoplasm of lip, oral cavity, and pharynx & $140 . x-149 . x$ \\
Malignant neoplasm of digestive organs and peritoneum & $150 . x-159 . x$ \\
Malignant neoplasm of respiratory and intrathoracic organs & $160 . x-165 . x x$ \\
Malignant neoplasm of bone, connective tissue, skin, and breast & $170 . x-176 . x$ \\
Malignant neoplasm of genitourinary organs & $179 . x-189 . x$ \\
Malignant neoplasm of other and unspecified sites & $190 . x-199 . x$ \\
Malignant neoplasm of lymphatic and hematopoietic tissue & $200 . x x-208 . x x$ \\
Carcinoma in situ & $230 . x-234 . x$ \\
\hline
\end{tabular}




\section{References}

1. The Facts About Inflammatory Bowel Diseases. Crohn's and Colitis Foundation of America. http://www.ccfa.org/assets/pdfs/ updatedibdfactbook.pdf. Accessed Apr 2017.

2. Molodecky NA, Soon S, Rabi DM, et al. Increasing incidence and prevalence of the inflammatory bowel diseases with time, based on systematic review. Gastroenterology. 2012;142(1):46-54.

3. John ES, Katz K, Saxena M, et al. Management of inflammatory bowel disease in the elderly. Curr Treat Options Gastroenterol. 2016;14(3):285-304.

4. Katz S, Pardi DS. Inflammatory bowel disease of the elderly: frequently asked questions (FAQs). Am J Gastroenterol. 2011;106(11):1889-97.

5. Charpentier C, Salleron J, Savoye G, et al. Natural history of elderly-onset inflammatory bowel disease: a population-based cohort study. Gut. 2014;63(3):423-32.

6. Juneja M, Baidoo L, Schwartz MB, et al. Geriatric inflammatory bowel disease: phenotypic presentation, treatment patterns, nutritional status, outcomes, and comorbidity. Dig Dis Sci. 2012;57(9):2408-15.

7. Johnson SL, Bartels CM, Palta M, et al. Biological and steroid use in relationship to quality measures in older patients with inflammatory bowel disease: a US Medicare cohort study. BMJ Open. 2015;5(9):e008597.

8. Stallmach A, Hagel S, Gharbi A, et al. Medical and surgical therapy of inflammatory bowel disease in the elderly-prospects and complications. J Crohn's Colitis. 2011;5(3):177-88.

9. Taleban S, Colombel JF, Mohler MJ, et al. Inflammatory bowel disease and the elderly: a review. J Crohn's Colitis. 2015;9(6):507-15.

10. Setoguchi S, Solomon DH, Glynn RJ, et al. Agreement of diagnosis and its date for hematologic malignancies and solid tumors between Medicare claims and cancer registry data. Cancer Causes Control. 2007;18(5):561-9.

11. Howlader N, Noone AM, Krapcho M, Miller D, Bishop K, Kosary CL, Yu M, Ruhl J, Tatalovich Z, Mariotto A, Lewis DR, Chen HS, Feuer EJ, Cronin KA (eds). SEER Cancer Statistics Review, 1975-2014, National Cancer Institute. Bethesda, MD. https://seer.cancer.gov/csr/1975_2014/, based on November 2016 SEER data submission, posted to the SEER web site, April 2017.

12. Hajjar ER, Cafiero AC, Hanlon JT. Polypharmacy in elderly patients. Am J Geriatr Pharmacother. 2007;5(4):345-51.
13. Lichtenstein GR, Hanauer SB, Sandborn WJ. Management of Crohn's disease in adults. Am J Gastroenterol. 2009;104(2):465-83.

14. Kornbluth A, Sachar DB. Ulcerative colitis practice guidelines in adults: American College of Gastroenterology, practice parameters committee. Am J Gastroenterol. 2010;105(3):501-23.

15. Gisbert JP, Chaparro M. Systematic review with meta-analysis: inflammatory bowel disease in the elderly. Aliment Pharmacol Ther. 2014;39(5):459-77.

16. Cross RK, Lapshin O, Finkelstein J. Patient subjective assessment of drug side effects in inflammatory bowel disease. J Clin Gastroenterol. 2008;42(3):244-51.

17. Osterman MT, Sandborn WJ, Colombel JF, et al. Increased risk of malignancy with adalimumab combination therapy, compared with monotherapy, for Crohn's disease. Gastroenterology. 2014;146(4):941-9.

18. Lichtenstein GR, Feagan BG, Cohen RD, et al. Drug therapies and the risk of malignancy in Crohn's disease: results from the TREAT $^{\mathrm{TM}}$ Registry. Am J Gastroenterol. 2014;109(2):212-23.

19. Nyboe AN, Pasternak B, Basit S, et al. Association between tumor necrosis factor- $\alpha$ antagonists and risk of cancer in patients with inflammatory bowel disease. JAMA. 2014;311:2406-13.

20. Beaugerie L, Carrat F, Colombel JF, et al. Risk of new or recurrent cancer under immunosuppressive therapy in patients with IBD and previous cancer. Gut. 2014;63(9):1416-23.

21. Lam MC, Bressler B. Vedolizumab for ulcerative colitis and Crohn's disease: results and implications of GEMINI studies. Immunotherapy. 2014;6(9):963-71.

22. Shelton E, Allegretti JR, Stevens B, et al. Efficacy of vedolizumab as induction therapy in refractory IBD patients: a multicenter cohort. Inflamm Bowel Dis. 2015;21(12):2879-85.

23. Löwenberg M, D’Haens G. Next-generation therapeutics for IBD. Curr Gastroenterol Rep. 2015;17(6):1-8.

24. Danese S, Vuitton L, Peyrin-Biroulet L. Biologic agents for IBD: practical insights. Nat Rev Gastroenterol Hepatol. 2015;12(9):537-45.

25. Cheddani H, Dauchet L, Fumery M, et al. Cancer in elderly onset inflammatory bowel disease: a population-based study. Am J Gastroenterol. 2016;111(10):1428-36.

26. Taleban S, Elquza E, Gower-Rousseau C, et al. Cancer and inflammatory bowel disease in the elderly. Dig Liver Dis. 2016;48(10):1105-11. 